\title{
Association of Skin Thickness With Alar Base Reduction in Patients Undergoing Lateral Crural Repositioning and Strut Grafting
}

A. Emre Ilhan, MD; Tevfik Sozen, MD; Basak Caypinar Eser, MD; Betul Cengiz, MD

IMPORTANCE Knowing the operation plan is important for rhinoplasty surgeons to prevent unpredictable results.

OBJECTIVES To investigate the frequency of alar base resection in patients with different skin thickness who underwent lateral crural repositioning and lateral crural strut graft and to evaluate the results in the context of the current literature.

DESIGN, SETTING, AND PARTICIPANTS This retrospective case series study included 621 patients who underwent primary open septorhinoplasty by the same surgeon between January 1, 2012, and June 30, 2015. From the surgical notes, operation type (lateral crural repositioning [LCrep] with lateral crural strut grafting [LCSG] and with or without alar base resection) and skin type were recorded. Study participants' skin types were determined intraoperatively and divided into 3 groups: (1) thick skin (the tip definition was limited by skin thickness and subcutaneous tissue), (2) thin skin (the tip cartilage was visible and could be observed despite overlying soft tissue and skin), and (3) normal skin (the tip cartilage during the procedure had no effect on the tip definition).

MAIN OUTCOMES AND MEASURES No primary study outcome was established before data collection began. The hypothesis was formulated during data collection.

RESULTS Of the total 621 patients in the study, 95 (15.3\%) were men and 526 (84.7\%) were women. Lateral crural repositioning with LCSG was performed in 319 surgical procedures (51.4\%), and alar base reduction was performed in 329 (53.0\%). The rate of alar base resection differed significantly on the basis of whether LCrep with LCSG was performed (odds ratio [OR], 1.821; 95\% Cl, 1.324-2.504; $P<.001$ ). In patients with thin skin, there was a marked difference in the incidence of alar base resection associated with LCrep with LCSG, but the difference was not statistically significant ( $\mathrm{OR}, 2.034 ; 95 \% \mathrm{Cl}, 0.912-4.539 ; P=.08$ ). In patients with thick skin, a significant difference in the frequency of alar base resection was associated with the application of LCrep with LCSG (OR, 1.995; 95\% Cl, 1.228-3.241; $P=.005)$. In patients with normal skin, LCrep with LCSG had no significant association with the frequency of alar base resection $(\mathrm{OR}, 1.557 ; 95 \% \mathrm{Cl}, 0.930-2.607 ; P=.09)$.

CONCLUSIONS AND RELEVANCE The necessity of alar base reduction after LCrep with LCSG is greater in patients with thick skin than in patients with thin and normal skin. This study is the first to examine this topic in rhinoplasty.

LEVEL OF EVIDENCE 3.
Author Affiliations: Rino Center, Istanbul, Turkey (Ilhan, Cengiz); Hacettepe University, Ankara, Turkey (Sozen); Special Ersoy Hospital, Istanbul, Turkey (Caypinar Eser). Corresponding Author: Basak Caypinar Eser, MD, Special Ersoy Hospital, Istanbul 34606, Turkey (bskcypnr@hotmail.com). 
$\mathrm{T}$ he most important stage of an aesthetic nose surgery is tip-plasty. It is not always possible to correct nasal tip deformities and positional anomalies in the cartilage structures that form the nasal tip using classic suture-andgraft techniques. Lateral crural repositioning (LCrep) with lateral crural strut grafting (LCSG) is one of the most advanced techniques in current aesthetic nasal tip surgery; it is an imperative method to master for surgeons who want excellent rhinoplasty outcomes. Patient selection for this procedure requires preoperative planning based on existing tip position problems.

Lateral crural repositioning with LCSG corrects tip asymmetry and malposition, especially in cases that cannot be repaired with traditional tip-plasty suturing, such as very thin, malpositioned, concave, or asymmetric tip cartilage. ${ }^{1,2}$ In addition, it corrects parentheses tip deformity and boxy nasal tip, which cannot be fixed using other methods. In recent years, LCrep with LCSG has become a common technique used in patients with cephalic positioning of the lateral crura. It was first described by Jack P. Gunter, who claimed it an effective solution for boxy nasal tip, malposition, alar rim retraction, alar rim collapse, and pathologic conditions of the lateral crura, such as concave lateral crura. ${ }^{3}$ Lateral crural repositioning eliminates length differences between nasal projection and the dorsum, defines projection by changing the dome position, and allows the liberation of the lateral crura and fixation to the medial crura at the desired position. ${ }^{1}$

However, every technique has advantages and disadvantages. One of the disadvantages of LCrep with LCSG is the possibility of postoperative problems, such as graft visibility and palpable hardness, especially in patients with thin skin. Furthermore, a graft of the incorrect length under the lateral crura can cause the area of the lateral crural complex insertion to protrude into the nasal cavity, occluding the external valve and the airway. Other disadvantages are that it requires extra cartilage, necessitates the elevation of the lateral crura from the underlying mucosa, and is technically challenging. Toriumi and Asher ${ }^{1}$ stated that, by increasing alar spread, LCrep with LCSG resulted in alar base widening and thus increased the frequency of alar base reduction. However, no studies have been conducted on this topic.

In this study, we investigated the frequency of alar base resection in patients with different skin types who underwent LCrep with LCSG, and we evaluated the results in the context of the current literature.

\section{Methods}

This retrospective case series included 621 patients who underwent primary open septorhinoplasty by one of us (A. E. I.) between January 1, 2012, and June 30, 2015, at the Rino Center, Istanbul, Turkey. Surgical records of the patients were analyzed retrospectively. From the surgical notes, operation type (LCrep with or without LCSG and with or without alar base resection) and skin type determined intraoperatively were re-

\section{Key Points}

Question Is there a significant difference in the frequency of alar base resection after primary rhinoplasty operations involving lateral crural repositioning with lateral crural strut grafting?

Findings In this study that included 621 patients who underwent primary open septorhinoplasty, female sex, application of lateral crural repositioning with lateral crural strut grafting, and thick skin were statistically significantly associated with alar base positivity.

Meaning The frequency of alar base reduction following lateral crural repositioning and lateral crural strut grafting is greater in patients with thick skin than in others with thin or normal skin. This study is the first to examine this topic in rhinoplasty.

corded. This study was approved by the ethical committee of Hacettepe University, and written patient informed consent was obtained.

Before surgery, all patients underwent a routine otorhinolaryngologic examination and were evaluated by endoscopic nasal examination using a 0-degree endoscope. Wide alar bases, wide sills, and alar flares were noted preoperatively and evaluated during the operation after the other steps of rhinoplasty. Patients with chronic sinusitis, nasal polyps, history of asthma or allergic rhinitis, and previous septoplasty or rhinoplasty were excluded from the study.

An angle of $30^{\circ}$ or less between the caudal edge of the lateral crura and the midline of the dorsum as measured by a gonioscope was considered cephalic malposition (Figures 1, 2, 3, and 4). ${ }^{4}$ Patients with such malposition underwent LCrep with LCSG. ${ }^{5}$ Afterward, tip sutures and columellar supporting grafts appropriate for the patient (tongue-in-groove or columellar strut) and cap grafts were applied to increase tip definition.

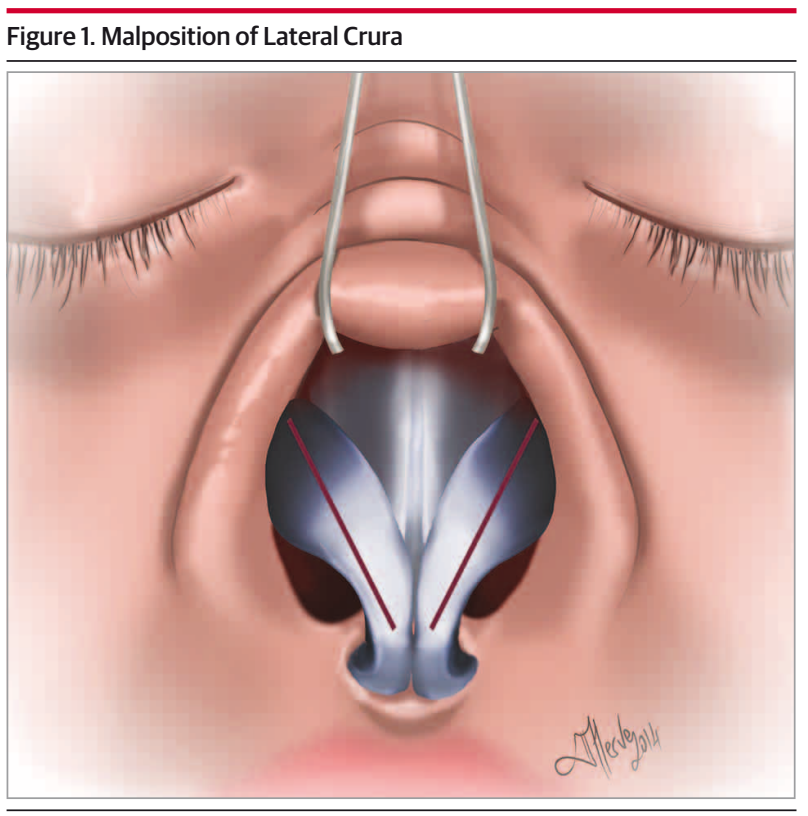

Measurement of angle between lateral crura and midline to confirm lateral crural malposition. 


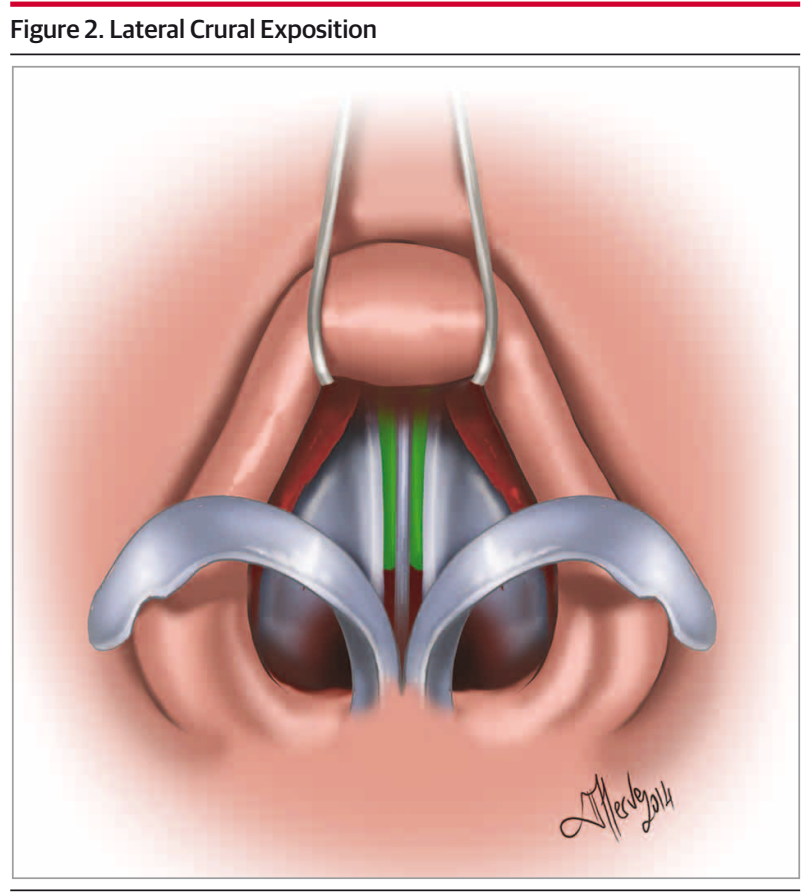

Lateral cartilages exposed by separation from their point of attachment to accessory cartilages.

How rhinoplasty maneuvers affected alar base width and flaring was identified using sequential photographs taken intraoperatively after each stage of rhinoplasty. The surgeon's interpretation of the intraoperative photographs was that skeletonization of the lateral crura, LCrep with LCSG increasing alar width, and tip suturing without lateral crural steal did not affect the width of the alar base, but cap grafting and lateral crural steal reduced alar width (eFigures 1-8 in the Supplement).

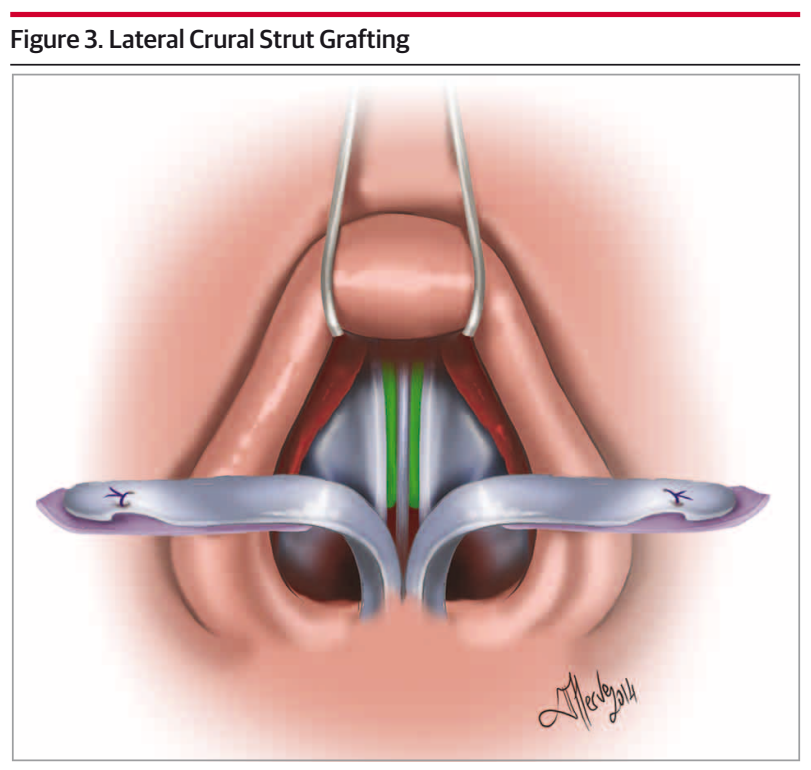

Shaped cartilage graft placed under lateral crural cartilage and sutured.

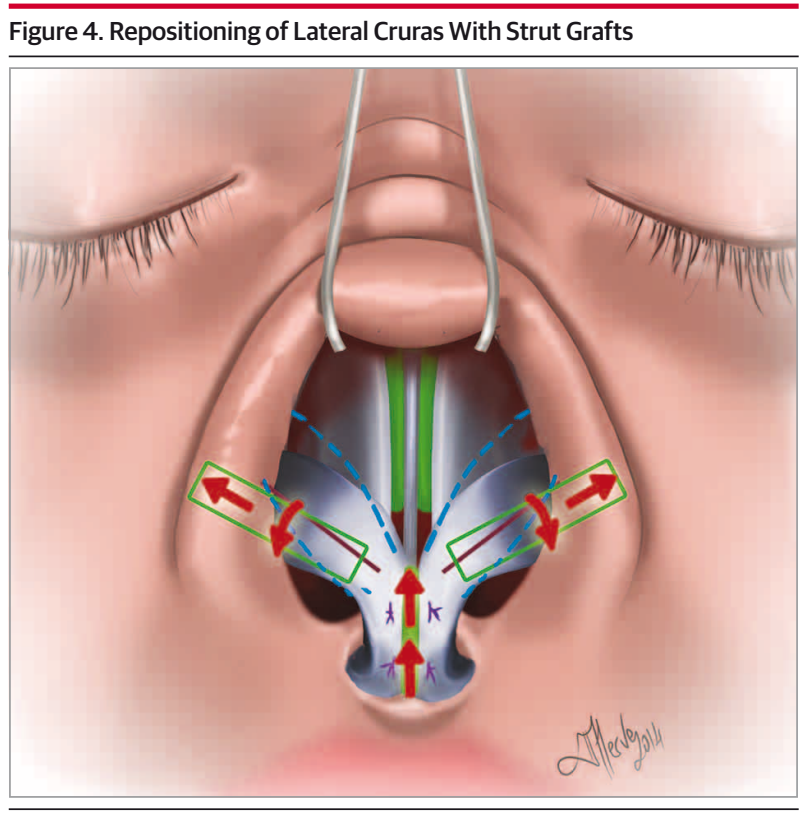

Positioning of lateral cruras in pockets formed on the anterior caudal region of the accessory cartilages.

Patients' skin types were determined intraoperatively and divided into 3 groups: (1) thick skin (the tip definition was limited by extra skin and subcutaneous tissue), (2) thin skin (the tip cartilage was visible and could be observed despite the overlying soft tissue), and (3) normal (the tip cartilage during the procedure had no effect on the tip definition). ${ }^{6}$

The nasal alae were evaluated at the conclusion of the procedure after suturing of the inverted $\mathrm{V}$ incision. Alar base resection was performed on patients with an alar base width exceeding the intercanthal distance and patients with increased alar spread or nasal flare. In the decision to perform alar base resection, the width of the nasal tip in proportion to the base was considered in patients with wide nasal tips. Base reduction was not performed on patients whose nasal tip would look relatively wider, especially patients with thick skin. If the alae were within an acceptable distance of the medial canthus line, the procedure was not performed and the natural structure of the nostrils was preserved. ${ }^{1}$

\section{Surgical Technique}

A closed approach using endonasal techniques may be preferable for rhinoplasty, but studies have demonstrated that an open-approach rhinoplasty is more suitable for patients undergoing repositioning. ${ }^{1}$ A standard 1:100 000 mixture of lidocaine, $1 \%$, and epinephrine was injected into the septum and outer nose as local anesthesia. The standard inverted V incision was made with a No. 11 blade, and bilateral marginal incisions were made with a No. 15 blade. Following skeletonization, the caudal septum was exposed gently. Hump resection was performed, after which the primary nasal dorsum height was determined. The septodorsal and bony-cartilaginous junction, or the keystone area, was shaped using a power rasp. The mucoperichondria were elevated bilaterally, and the graft was obtained. The septal L-strut was left to support the dorsum and 


\begin{tabular}{|c|c|c|c|c|}
\hline \multirow[b]{2}{*}{ Characteristic } & \multicolumn{4}{|l|}{ №. (\%) } \\
\hline & $\begin{array}{l}\text { Total } \\
(\mathrm{N}=621)\end{array}$ & $\begin{array}{l}2013 \\
(n=301)\end{array}$ & $\begin{array}{l}2014 \\
(n=220)\end{array}$ & $\begin{array}{l}2015 \\
(n=100)\end{array}$ \\
\hline \multicolumn{5}{|l|}{ Sex } \\
\hline Male & $95(15.3)$ & $55(18.3)$ & 28 (12.7) & $12(12.0)$ \\
\hline Female & $526(84.7)$ & $246(81.7)$ & $192(87.3)$ & $88(88.0)$ \\
\hline \multicolumn{5}{|c|}{ LCrep with LCSG } \\
\hline Negative $^{a}$ & $302(48.6)$ & $145(48.2)$ & $89(40.5)$ & $68(68.0)$ \\
\hline Positive & $319(51.4)$ & $156(51.8)$ & $131(59.5)$ & $32(32.0)$ \\
\hline \multicolumn{5}{|c|}{ Alar base resection } \\
\hline Negative $^{\mathrm{b}}$ & $292(47.0)$ & $144(47.8)$ & $98(44.5)$ & $50(50.0)$ \\
\hline Positive & $329(53.0)$ & $157(52.2)$ & $122(55.5)$ & $50(50.0)$ \\
\hline
\end{tabular}

Abbreviations: LCrep, lateral crural
repositioning; LCSG, lateral crural
strut grafting.
a Negative means LCrep with LCSG
has not been applied, while positive
means this maneuver has been
applied.
${ }^{\text {b }}$ Negative means alar base resection
has not been applied, while positive
means this maneuver has been
applied.

caudal. Before LCSG, a septal cartilage graft was obtained from each patient. Bony vault width and nasal bone spacing were evaluated in all patients, and medial or paramedian osteotomy was performed in selected patients, followed by high to low internal osteotomy for all patients. Asymmetric spreader grafts were placed to reconstruct the middle vault, followed by tip-plasty. Patients requiring repositioning were identified by measuring with a goniometer the angle between the lateral crura and the midline. Lateral crural repositioning with LCSG was performed on patients with an angle less than $30^{\circ}$ between the lateral crura and the midline, while the surgery proceeded directly to tip-plasty in patients with an angle of $30^{\circ}$ or more.

For patients undergoing LCrep with LCSG, the vestibular mucosa below the lower lateral cartilage was infiltrated with local anesthetic and hydrodissected and was then dissected from the caudal edge to the cephalic edge with iris scissors. The mucosal connection at the cephalic edge of the lateral cartilage was separated while leaving the cutaneous connection in the anterior caudal region intact. The lateral cartilages were freed by separating them from the accessory cartilages. Pieces of cartilage 3 to $4 \mathrm{~mm}$ wide and 15 to $25 \mathrm{~mm}$ long were removed from the septum and shaped for grafting. The graft was placed under the lateral cartilage with its tip extending $5 \mathrm{~mm}$ beyond the cephalic tip of the lateral crura and secured with a pair of 5-0 polyglactin 910 (Vicryl) sutures. Bilateral pockets were created anterior and caudal to the accessory cartilage dissecting the tissues in the direction of the lateral canthus, and the lateral crura supported by the lateral crural strut grafts were placed in these pockets in contact with the anterior nasal aperture. After the lateral crura and grafts were positioned in the pockets, the lateral crural strut grafts were fixed to the vestibular skin with 5-0 polyglactin 910 sutures (Figures 1-4).

Following middle vault modification in patients who did not undergo LCrep with LCSG, columellar supporting grafts were applied using the appropriate graft type to achieve tip definition, and the inverted V incision was sutured with 6-0 polypropylene (Prolene). After suturing, patients were assessed for alar base resection. If the interalar distance exceeded the intercanthal distance or alar flare was increased, marking was done for alar base resection using the midline as a reference. Starting from a midline at the columella, the excess nostril and alar amount was determined independently on both sides and triangular flaps were marked bilaterally on the medial alae, after which the alar crease was marked, extending at most to the 9-o'clock position on the right and the 3-o'clock position on the left. The excess skin was then marked and excised with a fullthickness incision on the natural crease using a No. 15 blade. After achieving hemostasis, the nasal alae were reinserted with 6-0 polypropylene sutures at close intervals. The columella was stabilized by placing bilateral fluted silicone tampons in the nasal passages. The operation was concluded by dressing the nose and applying a thermoplastic nasal splint.

\section{Statistical Analysis}

The NCSS (Number Cruncher Statistical System) 2007 software, version 07.1.9 (NCSS, LLC) was used for all statistical analyses. Descriptive statistical methods (mean, SD, frequency, and percentage) were used for quantitative and qualitative data accordingly. Three separate binary logistic regression analyses were performed to examine the association of sex, skin type, and application of LCrep with LCSG with the frequency of alar base resection. A multivariable logistic regression analysis was performed in which sex, skin type, LCrep with LCSG application, and all 2-way and 3-way interactions were independent variables and alar base resection was the dependent variable. Mantel-Haenszel tests were used to test the conditional independence of the association between LCrep with LCSG application and alar base resection at different levels of skin thickness. Two-sided $P \leq .05$ was accepted as statistically significant.

\section{Results}

Of the 621 patients in the study, 95 (15.3\%) were men and 526 (84.7\%) were women. Lateral crural repositioning with LCSG was performed in 319 surgical procedures (51.4\%), and alar base reduction was performed in 329 (53\%) (Table).

In the univariate analysis, sex, application of LCrep with LSCG, and skin thickness variables were introduced separately as independent variables while alar base resection was introduced as the dependent variable. Being a woman increased the risk of positive alar base resection by 2.061-fold (odds ratio [OR], 2.061; 95\% CI, 1.316-3.228; $P$ = .002). Appli- 
cation of LCrep with LSCG increased alar base resection positivity (OR, 1.821; 95\% CI, 1.324-2.504; $P<.001$ ). Skin thickness was not associated with alar base resection (OR, 1.325; 95\% CI, 0.934-1.879; $P$ > .11) (eTable in the Supplement).

We performed binary logistic regression analysis using the enter method to analyze the association of sex, skin type, and application of LCrep with LSCG with the frequency of alar base resection (eFigure 9 in the Supplement). Sex, skin type, LCrep with LCSG application, and all 2-way and 3-way interactions were introduced as independent variables while alar base resection was introduced as the dependent variable. None of the main effects and 2-way interactions was statistically significant. We found that being a woman (OR, 1.740; 95\% CI, 0.9623.149; $P=.07$ ), LCrep with LCSG application (OR, 1.784; 95\% CI, 0.986-3.229; $P=.06$ ), and thick skin (OR, 1.368; 95\% CI, $0.832-2.250 ; P=.02$ ) had a statistically significant association $(P=.02$; eFigure 10 in the Supplement). MantelHaenszel statistics were calculated for women and men separately. The effect of LCrep with LCSG application on alar base resection was analyzed at 3 different layers of skin thickness. Breslow-Day analysis was used to test the preliminary assumption of the homogeneity of the ORs and rejection for both men and women. Conditional independence was rejected for women $\left(X^{2}=10.821 ; P=.001\right)$. Rejection of conditional independence showed that the association between LCrep with LCSG application and alar base resection was significantly different for each skin thickness type. Fifty-one women (57.3\%) with normal skin who underwent LCrep with LCSG had alar base resection positivity compared with 39 thin-skinned women (58.2\%) and 83 thick-skinned women (68.6\%) who had the same procedure. The ORs of alar base resection positivity when LCrep with LCSG was applied compared with no LCrep with LCSG application were 1.289 (95\% CI, 0.726-2.291; $P=.39$ ) for normal skin, 1.990 (95\% CI, 0.861-4.600; $P=.11$ ) for thin skin, and 2.383 (95\% CI, 1.402-4.049; $P=.001$ ) for thick skin. These results showed that patients with thick skin and LCrep with LCSG application had greater risk of alar base positivity than patients with normal or thin skin (eFigures 11 and 12 and the eTable in the Supplement).

\section{Discussion}

Repositioning the lower lateral cartilages and then supporting them with lateral crural struts is one of the most advanced shaping methods in the tip-plasty stage of aesthetic rhinoplasty. Changing the angle of the lateral crura and supporting them from below with cartilage strut grafts allows the correction of a host of deformities, including boxy nasal tip, lateral crural malposition, alar retraction, nasal valve insufficiency, and concave lateral crura. ${ }^{3}$ The lateral crura is the principal anatomic structure forming the aesthetic and functional character of the nasal tip, making the LCrep with LCSG technique important. With the right techniques, it is possible to create an equilateral triangular nasal tip and functionally support the alar rims to prevent nasal obstruction. In lateral crural malposition, the angle between the midline and the lateral crural insertion point is $30^{\circ}$ or less. Studies indicate that malposition is one of the most common nasal tip deformities observed in patients undergoing primary and secondary rhinoplasty. ${ }^{7} \mathrm{~A}$ mathematical analysis of the effect of cephalic malposition on tip-plasty revealed differences in projection, rotation, and lateral crural length. Malposition has been found to affect tipplasty and lead to the development and use of different tipplasty techniques. ${ }^{8}$ Directing the lateral crural angle toward the medial canthus weakens the alae, resulting in parentheses tip deformity. ${ }^{9}$ New techniques have been attempted to correct this issue in noses with parentheses tip deformity and cephalic malposition. ${ }^{10}$

Toriumi and others ${ }^{11,12}$ reported that alar rim grafting was not necessary in patients undergoing LCrep and LCSG because those procedures provided sufficient support to the alar rims. Various techniques have been described to support and strengthen the lateral crura, such as alar batten grafting, but malposition cannot be solved by alar batten grafts alone and requires the combination of multiple approaches. Alar batten grafting may correct nasal valve insufficiency. ${ }^{12}$ However, this technique alone is not adequate for other aesthetic deformities, such as parentheses deformity and boxy nose.

The LCrep with LCSG method, although very effective, has disadvantages and advantages. These disadvantages include the dislocation of the lateral crural complex from the created pocket and the visibility of the lateral crural structures through the skin in the long term. In addition, because LCSGs do not extend to the anterior nasal aperture, they may protrude into the nasal cavity and occlude the airway. These problems usually occur if the surgeon is still mastering the technique or has made a mistake in patient selection. Further research into the patient selection criteria for the repositioning procedure is warranted. Separate analysis of patients with normal, thick, and thin skin who underwent LCrep with LCSG revealed no differences in aesthetic satisfaction or functional improvement according to skin type. ${ }^{4}$ Toriumi and Asher ${ }^{1}$ claimed that LCrep with LCSG has increased the frequency of alar base resection. Although repositioning is effective at reducing projection and providing support to the alar rims, it leads to alar flare or wider nasal base. In our study, the frequency of alar base resection was approximately 2-fold higher in patients who underwent LCrep with LCSG. Our results support those of Toriumi and Asher ${ }^{1}$ for patients of all skin types.

Procedures to narrow the alar base began to emerge more than a century ago. Alar base resection was first performed in 1892 by Robert Weir, who described the technique as an external wedge resection for the correction of unequal nostrils. ${ }^{13}$ In 1931, Joseph ${ }^{14}$ performed alar base resection by an internal excision, turning the alar base resection into an internal approach. Many investigators have refined the method over the years in an attempt to minimize the scarring that can form from the classic Weir incision. ${ }^{15-18}$

In our analysis of the skin type (normal, thin, or thick) of patients who underwent LCrep with LCSG, we found that alar base resection was performed considerably more often on patients with thick skin. Looking at patients of all skin types, we found that the frequency of alar base resection was higher in patients who underwent LCrep with LCSG. Our results support the findings by Toriumi and Asher. ${ }^{1}$ No substantial dif- 
ference was detected in patients with normal skin, but in patients with thin skin there was a marked but statistically nonsignificant trend toward a higher rate of alar base resection performed along with LCrep with LCSG. Surgeons planning to use these techniques should consider the patient's skin type preoperatively.

\section{Limitations}

We selected skin types intraoperatively and according to the observation of the surgeon. ${ }^{6}$ This process is a limitation of our study: we lacked an objective tool, such as an ultrasonographic measurement or biopsy pattern. However, the same experienced surgeon evaluated all patients as a standardized observation of skin thickness.

\section{Conclusions}

Cephalic malposition is a common problem observed in primary and secondary rhinoplasty that affects patient satisfaction both functionally and aesthetically. Of the various techniques reported in the literature, the most effective is LCrep with LCSG. The combination of LCrep with LCSG and alar base reduction is becoming more common. The necessity of alar base reduction after LCrep with LCSG is greater in patients with thick skin than in those with thin and normal skin. Surgeons using the technique should be aware of this possibility during their preoperative analyses and plan the operation accordingly.

\section{ARTICLE INFORMATION}

Accepted for Publication: February 5, 2017.

Published Online: July 27, 2017. doi:10.1001/jamafacial.2017.0486

Author Contributions: Dr Caypinar Eser had full access to all of the data in the study and takes responsibility for the integrity of the data and the accuracy of the data analysis.

Study concept and design: Ilhan, Sozen, Caypinar Eser.

Acquisition, analysis, or interpretation of data: Ilhan, Caypinar Eser, Cengiz.

Drafting of the manuscript: Sozen, Caypinar Eser, Cengiz.

Critical revision of the manuscript for important intellectual content: Ilhan, Caypinar Eser.

Statistical analysis: Cengiz.

Administrative, technical, or material support: Ilhan, Caypinar Eser, Cengiz.

Study supervision: Ilhan, Sozen.

Conflict of Interest Disclosures: None reported.

\section{REFERENCES}

1. Toriumi DM, Asher SA. Lateral crural repositioning for treatment of cephalic malposition. Facial Plast Surg Clin North Am. 2015;23(1):55-71.

2. Sheen JH. Aesthetic Rhinoplasty. St Louis, MO: Mosby-Year Book Inc; 1978.

3. Gunter JP, Friedman RM. Lateral crural strut graft: technique and clinical applications in rhinoplasty. Plast Reconstr Surg. 1997;99(4):943-952.
4. Ilhan AE, Saribas B, Caypinar B. Aesthetic and functional results of lateral crural repositioning. JAMA Facial Plast Surg. 2015;17(4):286-292.

5. Sheen JH, Sheen AP. Aesthetic Rhinoplasty. 2nd ed. St Louis, MO: Mosby; 1987.

6. Lavinsky-Wolff M, Dolci JE, Camargo HL Jr, et al. Vertical dome division: a quality-of-life outcome study. Otolaryngol Head Neck Surg. 2013;148(5): 758-763.

7. Constantian MB. The boxy nasal tip, the ball tip, and alar cartilage malposition: variations on a theme-a study in 200 consecutive primary and secondary rhinoplasty patients. Plast Reconstr Surg. 2005;116(1):268-281.

8. Sepehr A, Alexander AJ, Chauhan N, Chan H, Adamson PA. Cephalic positioning of the lateral crura: implications for nasal tip-plasty. Arch Facial Plast Surg. 2010;12(6):379-384.

9. Sheen JH. Malposition of alar cartilages. Paper presented at: American Society of Aesthetic Plastic Surgery Annual Meeting; May 1992; Los Angeles, CA.

10. Oktem F, Tellioğlu AT, Menevşe GT. Cartilage Z plasty on lateral crus for treatment of alar cartilage malposition. J Plast Reconstr Aesthet Surg. 2010;63 (5):801-808.

11. Toriumi DM. New concepts in nasal tip contouring. Arch Facial Plast Surg. 2006;8(3):156185.
12. Toriumi DM, Josen J, Weinberger M, Tardy ME Jr. Use of alar batten grafts for correction of nasal valve collapse. Arch Otolaryngol Head Neck Surg. 1997;123(8):802-808.

13. Weir RF. Restoring sunken noses without scarring the face. NY Med J. 1892;56:449-454.

14. Joseph J. Nasenplastik und sostige Gesichtsplastik nebst einem Anhang über Mamaplastik. Leipzig, Germany: Curt Koitzsch; 1931 110-113.

15. Guyuron B, Behmand RA. Alar base abnormalities: classification and correction. Clin Plast Surg. 1996;23(2):263-270.

16. Adamson PA, Oakley S, Tropper GJ, McGraw BL. Analysis of alar base narrowing. Am J Cosmet Surg. 1990;7(4):239-243.

17. Ribeiro $L$. The nasal ala in rhinoplasty-a new approach. Ann Plast Surg. 1981;6(6):448-452.

18. Watanabe K. New ideas to improve the shape of the ala of the Oriental nose. Aesthetic Plast Surg. 1994;18(4):337-344.

19. Rohrich RJ, Raniere J Jr, Ha RY. The alar contour graft: correction and prevention of alar rim deformities in rhinoplasty. Plast Reconstr Surg. 2002;109(7):2495-2505. 